\title{
Modifying effects of 1,2-dichloropropane on N-nitrosobis(2-oxopropyl)amine-induced cholangiocarcinogenesis in male Syrian hamsters
}

\author{
Min Gi, Masaki Fujioka, Shotaro Yamano, Eri Shimomura, Masayuki Kanki, \\ Satoko Kawachi, Hirokazu Tachibana, Kumiko Tatsumi, He Fang, Naomi Ishii, \\ Anna Kakehashi and Hideki Wanibuchi
}

Department of Molecular Pathology, Osaka City University Graduate School of Medicine, 1-4-3 Asahi-machi, Abeno-ku, Osaka 545-8585, Japan

(Received June 2, 2015; Accepted August 1, 2015)

\begin{abstract}
Based on the findings of epidemiological studies in Japan that occupational exposure to 1,2-dichloropropane (1,2-DCP) was associated with increased cholangiocarcinomas, 1,2-DCP has recently been classified as being carcinogenic to humans (Group 1). However, the cholangiocarcinogenicity of 1,2-DCP has not been demonstrated experimentally, and it was negative for cholangiocarcinogenicity in rats and mice. The present study determined the effects of 1,2-DCP on N-nitrosobis(2-oxopropyl)amine (BOP)-induced cholangiocarcinogenesis in male hamsters. We found that 1,2-DCP did not enhance the development of BOP-induced atypical biliary hyperplasia and did not induce any lesions in liver bile duct when administered alone. Notably, 1,2-DCP had no effect on the proliferative activity of bile duct epithelial cells regardless of BOP-initiation. These results demonstrate that 1,2-DCP lacks promoting effects on BOP-induced cholangiocarcinogenesis and suggest the possibility that 1,2-DCP is not cholangiocarcinogenic to the hamster in the present model. In addition, 1,2-DCP also lacks promoting effects on pancreatic, lung, and renal carcinogenesis. As the occurrence of occupational cholangiocarcinomas in Japan might be attributed to exposure to multiple chemicals, the results of the present study indicate that it will be necessary to determine the cholangiocarcinogenic effects of concurrent exposure of 1,2-DCP and the other halogen solvents to which workers with cholangiocarcinomas were exposed.
\end{abstract}

Key words: 1,2-dichloropropane, N-nitrosobis(2-oxopropyl)amine, Cholangiocarcinogenicity, GST-T1, CYP2E1, Hamster

\section{INTRODUCTION}

1,2-dichloropropane (1,2-DCP) is a chemical intermediate widely used in the production of chlorinated organic chemicals such as tetrachloroethylene and carbon tetrachloride, and it is also used as an oil and fat solvent in dry cleaning fluids and paint removers (Benbrahim-Tallaa et al., 2014; IARC, 2014). However, 1,2-DCP has recently been classified as being carcinogenic to humans (Group 1) by the International Agency for Research on Cancer. This was based on the findings of epidemiological studies in Japan that occupational exposure to paint strippers containing 1,2-DCP was associated with increased cholangiocarcinomas (Kumagai et al., 2013; Kubo et al., 2014; Kumagai, 2014). These reports raise serious concern about the risk of cancer in industrial workers chronically exposed to these haloalkanes.

Experimental demonstration of 1,2-DCP cholangiocarcinogenicity will provide an entry point into the establishment of animal models of 1,2-DCP-related occupational cholangiocarcinoma that will not only greatly facilitate our understanding of the carcinogenic mechanisms of this disease but also provide useful tools for the preclinical evaluation of potential therapeutic agents. However, 1,2DCP has not been demonstrated to be carcinogenic to the bile duct in experimental animals. 1,2-DCP induced hepatocellular carcinomas in mice in a gavage carcinogenicity study (NTP, 1986a) and induced nasal tumors in a rat inhalation carcinogenicity study (Umeda et al., 2010), but was negative in a rat gavage carcinogenicity study (NTP,

Correspondence: Hideki Wanibuchi (E-mail: wani@med.osaka-cu.ac.jp) 
1986a).

The hamster is a useful cholangiocarcinogenesis model (Imray et al., 1992; Thamavit et al., 1993; Blechacz and Gores, 2008; Takahashi et al., 2008). The carcinogenicity of 1,2-DCP in hamsters, however, has not yet been evaluated. There is an urgent need for data on the carcinogenicity of 1,2-DCP in hamsters. Our previous shortterm study demonstrated that (1) a high dose of 1,2-DCP induced centrilobular hepatocellular necrosis in hamsters, (2) CYP2E1 is possibly the key enzyme responsible for the hepatocytotoxicity of 1,2-DCP, and (3) GSH conjugation catalyzed by GST-T1 may exert a cytoprotective role in hepatocytes (Gi et al., 2015). Notably, while bile duct epithelial cells of hamsters are negative for GST-T1, bile duct epithelial cells of mice are positive for GST-T1 and 1,2-DCP is not carcinogenic to the bile duct epithelial cells of mice (NTP, 1986a), agreeing with the proposition that GST-T1 protects cells against the toxicity of 1,2-DCP. The fact that bile duct epithelial cells of hamsters are negative for GST-T1 suggests that the responses to the toxicity of 1,2-DCP in the bile duct epithelial cells of hamsters might differs from the response in mice.

$\mathrm{N}$-nitrosobis(2-oxopropyl)amine (BOP) is a wellknown multiorgan carcinogen in hamsters, inducing tumors in the pancreas, liver, lung, and kidneys (Pour et al., 1978; Furukawa et al., 2003; Takahashi et al., 2008, 2011; Okamura et al., 2013). The main purpose of the present study was to determine the modifying effects of 1,2-DCP on BOP-induced cholangiocarcinogenesis in male Syrian hamsters. We also determined the effects of 1,2-DCP on pancreatic, lung, and renal carcinogenesis, and the expression of CYP2E1 and GST-T1 in preneoplastic and neoplastic lesions in the liver and pancreas.

\section{MATERIALS AND METHODS}

\section{Chemicals}

BOP (CAS No.: 60599-38-4, purity > 99\%) was obtained from Gifu Pharmaceutical University, Gifu, Japan. 1,2-DCP (CAS No.: 78-87-5, purity > 98\%) was obtained from Wako Pure Chemical Industries, Ltd., Osaka, Japan. Corn oil was obtained from Nakarai Chemicals Co., Kyoto, Japan.

\section{Animals and Experimental design}

The experimental protocol was approved by the Institutional Animal Care and Use Committee of Osaka City University Graduate School of Medicine and conducted in accordance with the Guidelines for Proper Conduct of Animal Experiments (Science Council of Japan, 2006). A total of 87 male Syrian hamsters at 5 weeks of age were obtained from Japan SLC Inc., Hamamatsu, Japan. The Laboratory Animal Center of Osaka City University Graduate School of Medicine is accredited by the Center for the Accreditation of Laboratory Animal Care and Use (CALAC), Japan Health Sciences Foundation (JHSF). Animals were housed in polycarbonate cages (3 hamsters/cage) in experimental animal rooms with a targeted temperature of $22 \pm 3{ }^{\circ} \mathrm{C}$, relative humidity of $55 \pm 5 \%$, and a 12-hr light/dark cycle. CE2 basal pellet diet (Clea Japan, Inc., Tokyo, Japan) and tap water were available ad libitum throughout the study. Body weight, food and water consumption were measured weekly. All animals were acclimated to the animal room environment for 7 days before being used for experiments.

Hamsters at 6 weeks of age were randomized into 5 groups (Table 1). During the first week of the experiment, hamsters in groups 1 to 3 (24 animals per group) were injected subcutaneously with BOP four times (on days $1,3,5$, and 7) at a dose of $10 \mathrm{mg} / \mathrm{kg}$ body weight (b.w.) to initiate hepatobiliary and pancreatic carcinogenesis, and hamsters in groups 4 and 5 received vehicle $(0.9 \%$ saline $)$ in the same manner. One week after the last BOP or vehicle treatment, hamsters in the BOP initiation groups (groups 1-3) were administered 1,2-DCP by gavage at doses of 0 (corn oil vehicle), 62.5, and $125 \mathrm{mg} / \mathrm{kg}$ b.w., respectively, 5 days per week for 15 weeks ( 9 animals from each group) or 17 weeks (15 animals from each group). The highest dose of $125 \mathrm{mg} / \mathrm{kg}$ b.w. was based on the results of our previous 4-week study in which $125 \mathrm{mg} / \mathrm{kg}$ b.w. did not show apparent clinical toxicity whereas $250 \mathrm{mg} / \mathrm{kg}$ b.w. resulted in a high mortality rate (Gi et al., 2015). 1,2-DCP was dissolved in corn oil at target doses with an administration volume of $3 \mathrm{~mL} / \mathrm{kg}$ b.w. Animals in the non-initiation groups were administered $125 \mathrm{mg} / \mathrm{kg}$ b.w. 1,2-DCP (group 4; 9 animals) or corn oil vehicle (group 5; 6 animals) in the same manner for 17 weeks.

\section{Experimental procedure}

Seventeen-week experiment

As the incidence of cholangiocellular tumors was reported to be $5-50 \%$ at week 17 in hamsters receiving the same BOP initiation regimen as the present study (Takahashi et al., 2008; Okamura et al., 2013), 9 hamsters per BOP initiation group (groups 1-3) were scheduled for examination at the end of week 17. At sacrifice, hamsters were euthanized by inhalation of an overdose of isoflurane (Abbott Japan Co., Ltd., Tokyo, Japan) using a Small Animal Anesthetizer (MK-A110D, Muromachi Kikai Co., LTD., Tokyo, Japan) coupled with an Anesthetic Gas Scavenging System (MK-T 100E, Muromachi Kikai Co., 
Effect of 1,2-DCP on BOP-induced cholangiocarcinogenesis in hamsters

LTD.). At necropsy, livers were perfused with 4\% paraformaldehyde through the portal vein to preserve samples for molecular analysis and then excised. A total of 17 sections (five sections from the left lateral, three sections each from the left medial, right medial, right lateral, and caudate lobes) were cut and placed in 4\% paraformaldehyde. Pancreases were excised, pined out, fixed on corkboards and then placed in 10\% phosphate buffered formalin. After $24 \mathrm{hr}$ fixation, macroscopic lesions and at least 7 sections from each of the duodenal, gastric and splenic lobes of the pancreas (Takahashi et al., 1977) were cut out for examination. Following fixation, liver and pancreas tissues were embedded in paraffin and processed for histopathological examination and immunohistochemical analysis.

Preneoplastic biliary lesions in the livers were diagnosed as atypical biliary hyperplasia that were characterized by an irregular proliferation of bile duct with irregular lumina, multi-layers of atypical epithelial cells, with or without intestinal metaplasia (Kurashina et al., 1988; Nakanuma, 2012; Hailey et al., 2014).

Preneoplastic and neoplastic ductal lesions in the pancreas were diagnosed as atypical ductal hyperplasia and pancreatic ductal adenocarcinoma, respectively (Pour et al., 1981; Takahashi et al., 2008; Kitahashi et al., 2011; Okamura et al., 2013). Atypical ductal hyperplasias in the pancreas were characterized by proliferation of the duct epithelium with minimal to moderate cellular atypia, with or without mucous metaplasia. The typical pancreatic ductal adenocarcinoma had a duct-like structure accompanied by a dense fibrous stroma and exhibited invasive growth.

\section{Nineteen-week experiment}

As pancreatic ductal carcinomas were observed in groups treated with 1,2-DCP after BOP initiation but not in the group given BOP initiation alone in the 17-week experiment (Table 3), the remaining hamsters were examined at the end of week 19. Hamsters were euthanized by inhalation of an overdose of isoflurane, as described above. Livers were excised routinely after exsanguination via transection of the abdominal aorta, without $4 \%$ paraformaldehyde perfusion, and weighed. Livers and pancreases were processed for histopathological and immunohistochemical examination as described above for the 17-week experiment. Lungs were excised and inflated with $10 \%$ phosphate buffered formalin. After $24 \mathrm{hr}$ fixation, macroscopic lesions and 5 sections from each of the left, right caudal, right cranial, and right middle lobes of the lung were cut out and processed for histopathological examination. In addition, the kidneys (three transverse sections from each kidney), spleen (one transverse section), and common bile duct with surrounding tissue (four transverse sections) were also processed for histopathological examination.

\section{Immunohistochemical analysis}

Liver and pancreas specimens containing preneoplastic or neoplastic lesions, 5 specimens each from the $125 \mathrm{mg} / \mathrm{kg}$ b.w. 1,2-DCP group (group 4) and the vehicle control group (group 5) in the 19-week experiment, and pancreas specimens containing neoplastic lesions from the 17-week experiment were examined for expression of CYP2E1, GST-T1, and Ki-67 by immunohistochemical staining using the avidin-biotin-peroxidase complex (ABC) method. Antigen retrieval was performed by microwaving at $98^{\circ} \mathrm{C}$ for $20 \mathrm{~min}$ in $0.01 \mathrm{M}$ citrate buffer ( $\mathrm{pH} 6.0$ ), endogenous peroxidase activity was blocked with $0.3 \% \mathrm{H}_{2} \mathrm{O}_{2}$ in distilled water for $5 \mathrm{~min}$, non-specific binding was blocked with normal goat serum at $37^{\circ} \mathrm{C}$ for $30 \mathrm{~min}$, and sections were then incubated with rabbit polyclonal anti-CYP2E1 antibody (ab28146, Abcam, Cambridge, MA, USA) at a dilution of 1:100, rabbit polyclonal anti-GST-T1 antibody (15838-1-AP, Proteintech Group, Inc., Chicago, IL, USA) at a dilution of 1:200, or rabbit monoclonal anti-Ki-67 antibody (\#4203-1, Epitomics, CA, USA) at a dilution of 1:500 overnight at $4^{\circ} \mathrm{C}$. Immunoreactivity was detected using a VECSTAIN Elite ABC Kit (Rabbit IgG) (PK-6101, Vector Laboratories, Burlingame, CA, USA) and 3,3'-diaminobenzidine hydrochloride (Sigma Chemical Co., St Louis, MO, USA). Hepatocytes are positive for CYP2E1, GST-T1, and $\mathrm{Ki}-67$, and therefore served as an internal positive control for immunohistochemical staining of hepatobiliary epithelium. A liver specimen served as the positive control for immunohistochemical staining of the pancreas and was included with each staining procedure. Rabbit IgG (I-1000, Vector Laboratories, Inc.) instead of the primary antibody served as the negative control and was included with each staining procedure.

\section{Statistical analysis}

All mean values are reported as mean \pm S.D. Statistical analyses were performed using the GraphPad Prism 6 program (GraphPad Software, Inc., CA, USA). Homogeneity of variance was tested by the F-test (two-group comparisons) or Bartlett test (multi-group comparisons). Differences in mean values between the vehicle control and $125 \mathrm{mg} / \mathrm{kg}$ alone treatment groups in the 19-week experiment were evaluated by Student's t-test when the variance was homogeneous and Welch's t-test when the variance was heterogeneous. Differences in mean val- 
ues between the group given BOP-initiation alone and the groups treated with 1,2-DCP after BOP initiation in the 17- and 19-week experiments were evaluated by the 2-tailed Dunnett test when variance was homogeneous and the Kruskal-Wallis non-parametric ANOVA followed by Dunn's test when the variance was heterogeneous. Differences in the incidence of liver and pancreas lesions between the control and treatment groups were evaluated by Fisher's exact test. $\mathrm{p}$ values less than 0.05 were considered significant.

\section{RESULTS}

\section{Body weights, liver weights, and average food and water consumption}

Body weights, liver weights, and average food and water consumption are summarized in Table 1. One hamster in the group treated with $62.5 \mathrm{mg} / \mathrm{kg}$ b.w. 1,2-DCP after BOP initiation (group 2 in the 19-week experiment) died of unknown reasons at week 12 and was not included in the analyses. All the remaining animals survived to the scheduled sacrifice.

In the 17-week experiment, final body weights were significantly decreased in the group treated with $125 \mathrm{mg} / \mathrm{kg}$ b.w. 1,2-DCP after BOP initiation (group 3) compared with the group given BOP alone (group 1). Food and water consumption tended to decrease in group 3 compared with group 1 .

At the end of week 19, similarly to the findings in the 17-week study, final body weights were significantly decreased, along with lower food and water consumption in the group treated with $125 \mathrm{mg} / \mathrm{kg} \mathrm{b.w.} \mathrm{1,2-}$ DCP after BOP initiation (group 3) compared with the group given BOP alone (group 1). Decreased final body weights and lower food consumption were also observed in the group treated with $125 \mathrm{mg} / \mathrm{kg}$ b.w. 1,2-DCP alone (group 4), albeit without statistical significance compared with the vehicle control group (group 5). Absolute, but not relative, liver weights tended to decrease in the hamsters treated with $125 \mathrm{mg} / \mathrm{kg}$ b.w. 1,2-DCP, regardless of BOP initiation, compared to their respective concurrent controls, possibly due to the lower body weights in the hamsters treated with $125 \mathrm{mg} / \mathrm{kg}$ b.w. 1,2-DCP.

\section{Histopathological findings in livers}

The histopathological findings in the livers are summarized in Table 2. There were no significant differences in incidence or multiplicity of atypical biliary hyperplasia (Figs. 1A and 1B) in livers between the groups treated with 1,2-DCP after BOP initiation and the group given BOP alone in the 17-week and 19-week experiments. 1,2-DCP treatment also had no effect on the development of cholangioma; one cholangioma was observed in the group treated with $62.5 \mathrm{mg} / \mathrm{kg}$ b.w. 1,2-DCP after BOP initiation in the 19-week experiment (Fig. 1C) but not in any other group.

There were no significant differences in the incidence or multiplicity of hepatocellular adenomas between the groups treated with 1,2-DCP after BOP initiation and the groups treated with BOP alone in the 17 -week and 19-week experiments. No atypical biliary hyperplasia, cholangioma or hepatocellular adenoma was observed in the group treated with 1,2-DCP alone in the 19-week experiment.

Table 1. Body weights, liver weights, and average food and water consumption.

\begin{tabular}{|c|c|c|c|c|c|c|c|c|c|}
\hline Group & $\begin{array}{l}\text { BOP } \\
\text { initiation }\end{array}$ & $\begin{array}{c}\text { 1,2-DCP } \\
\text { (mg/kg b.w.) }\end{array}$ & $\begin{array}{l}\text { Initial no. } \\
\text { of animals }\end{array}$ & $\begin{array}{l}\text { Effective no. } \\
\text { of animals }\end{array}$ & $\begin{array}{l}\text { Final body } \\
\text { weight (g) }\end{array}$ & $\begin{array}{c}\text { Absolute liver } \\
\text { weight }(\mathrm{g})\end{array}$ & $\begin{array}{l}\text { Relative liver } \\
\text { weight }(\%)\end{array}$ & $\begin{array}{l}\text { Average water } \\
\text { consumption } \\
\text { (g/day/hamster) }\end{array}$ & $\begin{array}{l}\text { Average food } \\
\text { consumption } \\
\text { (g/day/hamster) }\end{array}$ \\
\hline \multicolumn{10}{|c|}{ Seventeen-week experiment } \\
\hline 1 & + & 0 & 9 & 9 & $168 \pm 14$ & N.D. & N.D. & 8.1 & 7.3 \\
\hline 2 & + & 62.5 & 9 & 9 & $173 \pm 11$ & N.D. & N.D. & 8.1 & 7.3 \\
\hline 3 & + & 125 & 9 & 9 & $146 \pm 12^{*}$ & N.D. & N.D. & 7.5 & 6.7 \\
\hline \multicolumn{10}{|c|}{ Nineteen-week experiment } \\
\hline 1 & + & 0 & 15 & 15 & $170 \pm 9$ & $8.1 \pm 0.7$ & $4.8 \pm 0.3$ & 7.8 & 7.3 \\
\hline 2 & + & 62.5 & 15 & $14^{\mathrm{a}}$ & $173 \pm 12$ & $8.2 \pm 1.1$ & $4.7 \pm 0.4$ & 8.1 & 7.4 \\
\hline 3 & + & 125 & 15 & 15 & $155 \pm 12^{*}$ & $7.4 \pm 0.8$ & $4.7 \pm 0.3$ & 7.8 & 6.8 \\
\hline 4 & - & 125 & 9 & 9 & $166 \pm 14$ & $7.7 \pm 0.9$ & $4.6 \pm 0.2$ & 7.5 & 7.4 \\
\hline$\underline{5}$ & - & 0 & 6 & 6 & $181 \pm 13$ & $8.5 \pm 1.1$ & $4.7 \pm 0.4$ & 7.8 & 7.6 \\
\hline
\end{tabular}

N.D. not determined

a one hamster died at week 12 and was not included in the analyses.

$* \mathrm{p}<0,01$ vs. respective BOP alone treatment group. 
Effect of 1,2-DCP on BOP-induced cholangiocarcinogenesis in hamsters

Table 2. Histopathological findings in livers.

\begin{tabular}{|c|c|c|c|c|c|c|c|c|c|}
\hline \multirow{3}{*}{ Group } & \multirow{3}{*}{$\begin{array}{c}\mathrm{BOP} \\
\text { initiation }\end{array}$} & \multirow{3}{*}{$\begin{array}{c}\text { 1,2-DCP } \\
\text { (mg/kg b.w.) }\end{array}$} & \multirow{3}{*}{$\begin{array}{c}\text { Effective no. } \\
\text { of animals }\end{array}$} & \multicolumn{4}{|c|}{ Cholangiocellular lesions } & \multirow{2}{*}{\multicolumn{2}{|c|}{$\begin{array}{l}\text { Hepatocellular lesions } \\
\text { Hepatocellular adenoma }\end{array}$}} \\
\hline & & & & \multicolumn{2}{|c|}{ Atypical biliary hyperplasia } & \multicolumn{2}{|c|}{ Cholangioma } & & \\
\hline & & & & $\begin{array}{l}\text { Incidence } \\
(\%)\end{array}$ & $\begin{array}{l}\text { Multiplicity } \\
\text { (No./hamster) }\end{array}$ & $\begin{array}{l}\text { Incidence } \\
(\%)\end{array}$ & $\begin{array}{l}\text { Multiplicity } \\
\text { (No./hamster) }\end{array}$ & $\begin{array}{l}\text { Incidence } \\
(\%)\end{array}$ & $\begin{array}{l}\text { Multiplicity } \\
\text { (No./hamster) }\end{array}$ \\
\hline \multicolumn{10}{|c|}{ Seventeen-week experiment } \\
\hline 1 & + & 0 & 9 & $2(22.2 \%)$ & $0.44 \pm 0.88$ & 0 & 0 & 0 & 0 \\
\hline 2 & + & 62.5 & 9 & $3(33.3 \%)$ & $0.78 \pm 1.20$ & 0 & 0 & $1(11.1 \%)$ & $0.11 \pm 0.33$ \\
\hline 3 & + & 125 & 9 & $1(11.1 \%)$ & $0.11 \pm 0.33$ & 0 & 0 & $1(11.1 \%)$ & $0.11 \pm 0.33$ \\
\hline \multicolumn{10}{|c|}{ Nineteen-week experiment } \\
\hline 1 & + & 0 & 15 & $7(46.7 \%)$ & $0.80 \pm 0.94$ & 0 & 0 & $1(6.6 \%)$ & $0.07 \pm 0.26$ \\
\hline 2 & + & 62.5 & $14^{\mathrm{a}}$ & $6(42.9 \%)$ & $0.93 \pm 1.44$ & $1(7.1 \%)$ & $0.07 \pm 0.27$ & $1(7.1 \%)$ & $0.07 \pm 0.27$ \\
\hline 3 & + & 125 & 15 & $8(53.3 \%)$ & $0.73 \pm 1.02$ & 0 & 0 & 0 & 0 \\
\hline 4 & - & 125 & 9 & 0 & 0 & 0 & 0 & 0 & 0 \\
\hline 5 & - & 0 & 6 & 0 & 0 & 0 & 0 & 0 & 0 \\
\hline
\end{tabular}

a one hamster died at week 12 and was not included in the analyses.

Table 3. Histopathological findings in pancreases.

\begin{tabular}{|c|c|c|c|c|c|c|c|}
\hline \multirow[b]{2}{*}{ Group } & \multirow[b]{2}{*}{$\begin{array}{c}\text { BOP } \\
\text { initiation }\end{array}$} & \multirow[b]{2}{*}{$\begin{array}{c}\text { 1,2-DCP } \\
\text { (mg/kg b.w.) }\end{array}$} & \multirow[b]{2}{*}{$\begin{array}{c}\text { Effective no. } \\
\text { of animals }\end{array}$} & \multicolumn{2}{|c|}{ Atypical ductal hyperplasia } & \multicolumn{2}{|c|}{ Pancreatic ductal carcinoma } \\
\hline & & & & $\begin{array}{l}\text { Incidence } \\
(\%)\end{array}$ & $\begin{array}{l}\text { Multiplicity } \\
\text { (No./hamster) }\end{array}$ & $\begin{array}{l}\text { Incidence } \\
(\%)\end{array}$ & $\begin{array}{c}\text { Multiplicity } \\
\text { (No./hamster) }\end{array}$ \\
\hline \multicolumn{8}{|c|}{ Seventeen-week experiment } \\
\hline 1 & + & 0 & 9 & $4(44.4 \%)$ & $0.77 \pm 1.09$ & 0 & 0 \\
\hline 2 & + & 62.5 & 9 & $4(44.4 \%)$ & $0.67 \pm 0.87$ & $1(11.1 \%)$ & $0.11 \pm 0.33$ \\
\hline 3 & + & 125 & 9 & $4(44.4 \%)$ & $0.44 \pm 0.53$ & $3(33.3 \%)$ & $0.44 \pm 0.73$ \\
\hline \multicolumn{8}{|c|}{ Nineteen-week experiment } \\
\hline 1 & + & 0 & 15 & $10(66.7 \%)$ & $1.33 \pm 1.50$ & $5(33.3 \%)$ & $0.33 \pm 0.49$ \\
\hline 2 & + & 62.5 & $14^{\mathrm{a}}$ & $8(57.1 \%)$ & $0.79 \pm 0.89$ & $4(28.6 \%)$ & $0.29 \pm 0.46$ \\
\hline 3 & + & 125 & 15 & $8(53.3 \%)$ & $0.67 \pm 0.82$ & $4(26.7 \%)$ & $0.27 \pm 0.46$ \\
\hline 4 & - & 125 & 9 & 0 & 0 & 0 & 0 \\
\hline 5 & - & 0 & 6 & 0 & 0 & 0 & 0 \\
\hline
\end{tabular}

a one hamster died at week 12 and was not included in the analyses.

\section{Histopathological findings in pancreases}

The incidence and multiplicity of atypical ductal hyperplasia (Fig. 1D) and pancreatic ductal carcinoma (Figs. 1E and 1F) in pancreases are summarized in Table 3. In the 17-week experiment, the incidence of pancreatic ductal adenocarcinoma showed a tendency to increase in the group treated with $125 \mathrm{mg} / \mathrm{kg}$ b.w. 1,2DCP after BOP initiation $(3 / 9,33.3 \%)$ compared with the group given BOP alone $(0 / 9,0 \%)$. The incidence and multiplicity of atypical ductal hyperplasia were comparable between these two groups. In the 19-week experiment, however, there were no significant differences in the incidence or multiplicity of pancreatic ductal adenocarcinomas between the group treated with BOP alone $(5 / 15$, $33.3 \%$; $0.33 /$ hamster) and the groups treated with 62.5 $(4 / 14,28.6 \% ; 0.29 /$ hamster $)$ or $125 \mathrm{mg} / \mathrm{kg}$ b.w. 1,2-DCP $(4 / 15,26.7 \% ; 0.27 /$ hamster $)$ after BOP initiation. In addition, there were no significant differences in the incidence or multiplicity of atypical ductal hyperplasia among the BOP initiation groups.

Considering that the influence of the two weeks period between weeks 17 and 19 on the development of adenocarcinoma is likely to be limited, we also compared the total incidence and multiplicity of pancreatic ductal adenocarcinomas observed at weeks 17 and 19. Similar to the results at week 19, neither the total incidence nor the total multiplicity of adenocarcinoma significantly differed between the BOP initiation alone group (5/24, $20.8 \% ; 0.21 /$ hamster) and the groups treated with 62.5 $(5 / 23,21.7 \% ; 0.22 /$ hamster $)$ or $125 \mathrm{mg} / \mathrm{kg} \mathrm{b.w.} \mathrm{1,2-DCP}$ (7/25, 28\%; 0.33/hamster) after BOP initiation.

\section{Histopathological findings in lung and kidneys in the 19-week experiment}

There were no significant differences in the incidence or multiplicity of lung tumors between the groups treat- 

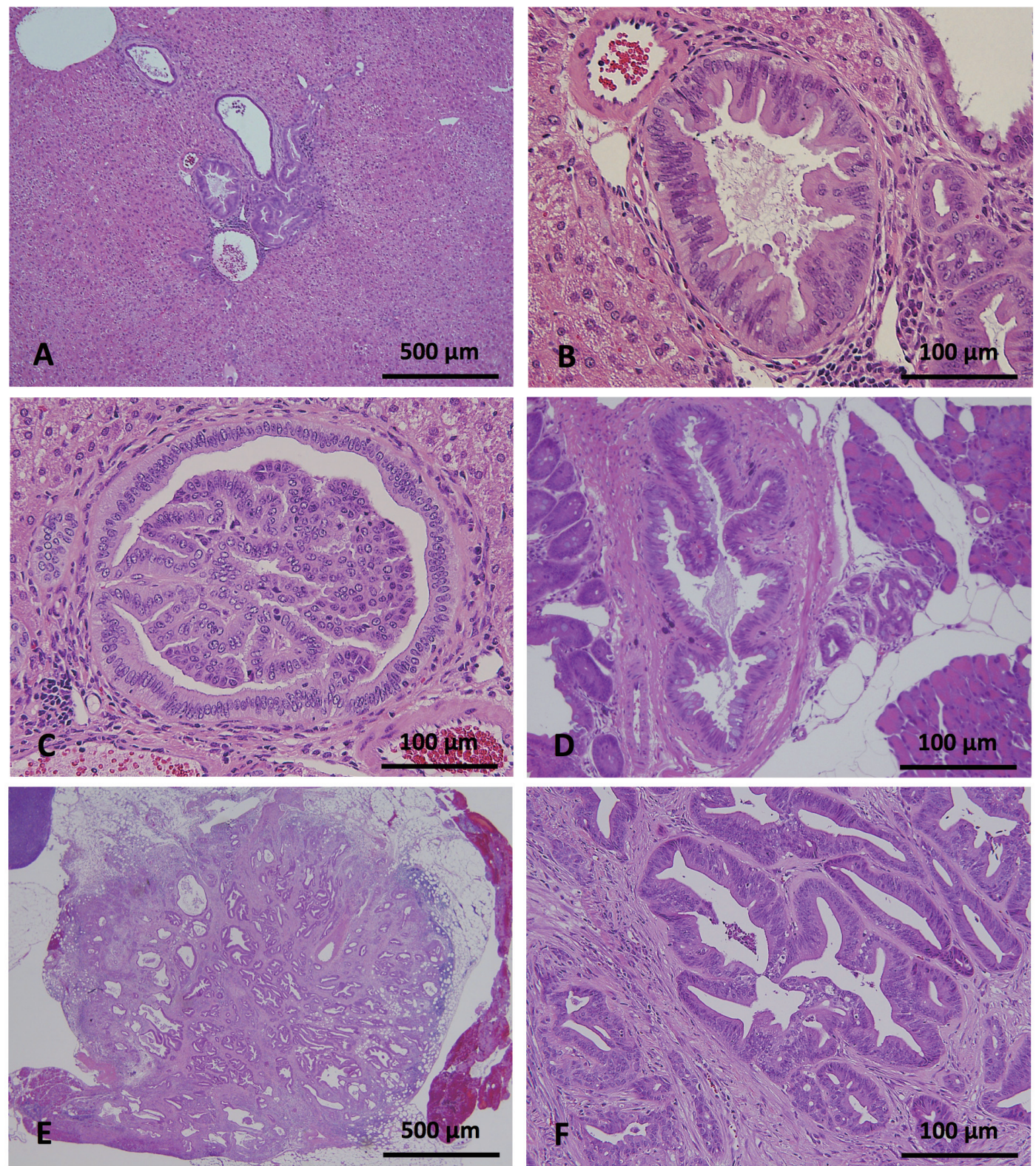

Fig. 1. Preneoplastic lesions and tumors in livers and pancreases in the 19-week experiment. (A) An atypical biliary hyperplasia in the liver of a hamster treated with $125 \mathrm{mg} / \mathrm{kg}$ b.w. 1,2-DCP after BOP initiation. (B) Higher magnification of A. (C) Cholangioma in a hamster treated with $62.5 \mathrm{mg} / \mathrm{kg}$ b.w. 1,2-DCP after BOP initiation. (D) An atypical ductal hyperplasia in the pancreas of a hamster treated with $125 \mathrm{mg} / \mathrm{kg}$ b.w. 1,2-DCP after BOP initiation. (E) Pancreatic ductal carcinoma in a hamster treated with BOP alone. (F) Higher magnification of E.

ed with 1,2-DCP after BOP initiation and the group given BOP alone (Table 4). One renal cell tumor was observed in the group treated with $62.5 \mathrm{mg} / \mathrm{kg}$ b.w. after BOP initiation but not in the other groups. No lung or kidney tumors were observed in the group treated with $125 \mathrm{mg} / \mathrm{kg}$ b.w. 1,2-DCP alone (group 4).

\section{Proliferative activity in livers and pancreases in the 19-week experiment}

There were no significant differences in the Ki-67 index in the atypical biliary hyperplasias in the livers (Fig. 2A) or atypical ductal hyperplasias in the pancreases (Fig. 2B) between the group given BOP alone and 
Effect of 1,2-DCP on BOP-induced cholangiocarcinogenesis in hamsters
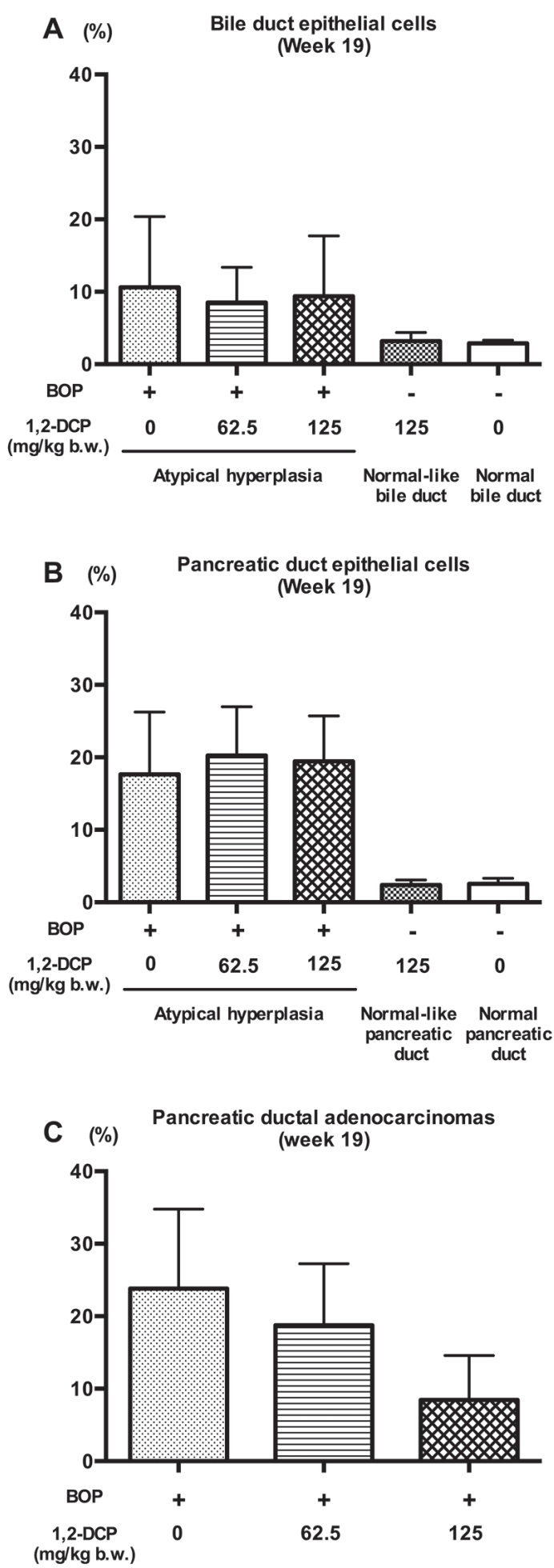

Fig. 2. Ki-67 index in biliary and pancreatic ducts in the 19week experiment. (A) Bile duct epithelium in livers. (B) Pancreatic duct epithelium. (C) Pancreatic ductal adenocarcinomas. the groups treated with 62.5 or $125 \mathrm{mg} / \mathrm{kg}$ 1,2-DCP after BOP initiation in the 19-week experiment. There were also no significant differences in the Ki-67 index in the bile duct epithelium (Fig. 2A) or pancreatic duct epithelium (Fig. 2B) between the vehicle control group and the group treated with $125 \mathrm{mg} / \mathrm{kg}$ b.w. 1,2-DCP alone. Moreover, in the 19-week experiment, the Ki-67 index in the pancreatic ductal adenocarcinomas did not differ significantly between the groups receiving 1,2-DCP after BOP initiation and the group given BOP alone (Fig. 2C). The $\mathrm{Ki}-67$ index in pancreatic ductal adenocarcinomas in the 17-week experiment could not be compared as no cancers were observed in the group given BOP alone. It should be noted, however, that there were no significant differences in the Ki-67 index in pancreatic ductal adenocarcinomas between the groups receiving 1,2-DCP after BOP initiation and the group given BOP alone when all the pancreatic ductal adenocarcinomas in the 17 and 19-week experiments were compared (Supplemental Fig. 1).

\section{GST-T1 and CYP2E1 immunohistochemistry}

Expression of CYP2E1 and GST-T1 was not observed in normal bile ducts or atypical biliary hyperplasias in the livers or normal pancreatic ducts, atypical ductal hyperplasias, or pancreatic ductal adenocarcinomas in the pancreases regardless of BOP and/or 1,2-DCP treatment.

\section{DISCUSSION}

While a specific animal model capable of predicting cholangiocarcinogenicity of chemicals in humans has not been established, the present BOP-induced multi-organ hamster carcinogenesis model is well established as a bioassay for evaluating the effects of chemicals in multiple organs/tissues including hepatobiliary system and pancreas (Pour et al., 1978; Furukawa et al., 2003; Takahashi et al., 2008, 2011; Okamura et al., 2013). To the best of our knowledge, this is the first study to determine the carcinogenic effects of 1,2-DCP in hamsters. Results of our thorough histopathological examination of livers (17 sections of liver per animal) show that 1,2-DCP did not enhance the development of BOP-induced atypical biliary hyperplasia that is associated with development of bile duct cancer (Kurashina et al., 1988; Nakanuma, 2012; Hailey et al., 2014) and did not induce any lesions in liver bile ducts when administered alone to hamsters. Notably, 1,2-DCP had no effect on the proliferative activity of bile duct epithelial cells regardless of BOP-initiation. These results indicate that 1,2-DCP lacks a promoting effect on BOP-induced cholangiocarcinogenesis in hamsters. Carcinogens (also referred to as com- 
Table 4. Histopathological findings in lungs.

\begin{tabular}{|c|c|c|c|c|}
\hline \multirow{2}{*}{$\begin{array}{l}\text { BOP } \\
\text { initiation }\end{array}$} & \multirow[b]{2}{*}{$\begin{array}{c}\text { 1,2-DCP } \\
\text { (mg/kg b.w.) }\end{array}$} & \multirow[b]{2}{*}{$\begin{array}{l}\text { Effective no. } \\
\text { of animals }\end{array}$} & \multicolumn{2}{|c|}{ Adenoma } \\
\hline & & & $\begin{array}{c}\text { Incidence } \\
(\%)\end{array}$ & $\begin{array}{c}\text { Multiplicity } \\
\text { (No./hamster) }\end{array}$ \\
\hline \multicolumn{5}{|c|}{ Nineteen-week experiment } \\
\hline+ & 0 & 15 & $4(26.7 \%)$ & $0.33 \pm 0.62$ \\
\hline+ & 62.5 & $14^{\mathrm{a}}$ & $4(28.6 \%)$ & $0.36 \pm 0.63$ \\
\hline+ & 125 & 15 & $5(33.3 \%)$ & $0.53 \pm 0.92$ \\
\hline- & 125 & 9 & 0 & 0 \\
\hline- & 0 & 6 & 0 & 0 \\
\hline
\end{tabular}

${ }^{\mathrm{a}}$ one hamster died at week 12 and was not included in the analyses.

plete carcinogens) have the ability to function at the initiation, promotion, and progression stages of carcinogenesis (Klaunig and Kamendulis, 2008). Therefore, the results of the present study suggest that 1,2-DCP is not cholangiocarcinogenic to the hamster in the present model. As the maximum tolerated dose of 1,2-DCP applicable to longterm hamster carcinogenicity studies may be between 125 (the highest dose in the present study) and $250 \mathrm{mg} /$ kg b.w. (Gi et al., 2015), further studies are warranted to determine the carcinogenic effects of higher doses of 1,2DCP in hamsters. In addition, given that the occupational cholangiocarcinomas that occurred in the Japanese off-set printing plants were associated with inhalation exposure to 1,2-DCP, inhalation carcinogenicity studies are also needed to clarify the cholangiocarcinogenic potential of 1,2 -DCP in hamsters.

It remains unclear whether the occupational cholangiocarcinoma that occurred in Japan can be attributed to 1,2-DCP alone as workers with cholangiocarcinoma were exposed to more than 20 different chemicals (BenbrahimTallaa et al., 2014; Sobue et al., 2015). Dichloromethane (DCM) is another haloalkane associated with the occupational cholangiocarcinoma cases and was therefore classified as probably carcinogenic to human (Group 2A) (Benbrahim-Tallaa et al., 2014; Sobue et al., 2015). In terms of cholangiocarcinogenicity in experimental rodents, similarly to 1,2-DCP, DCM did not induce cholangiocarcinogenesis in inhalation or drinking water carcinogenicity studies in mice, rats, or hamsters (Burek et al., 1984; Serota et al., 1986a, 1986b), while it induced hepatocellular tumors and lung tumors in inhalation carcinogenicity studies in mice (NTP, 1986b). Given the fact that so far there is no experimental evidence for 1,2-DCP or DCM cholangiocarcinogenicity when administered alone and the possibility that the occurrence of occupational cholangiocarcinomas in Japan might be attributed to exposure to multiple chemicals (Benbrahim-Tallaa et al., 2014; Sobue et al., 2015), studies are needed to deter- mine the carcinogenic effects of concurrent exposure to 1,2-DCP and DCM or other organic solvents contained in the stripers to which the workers with cholangiocarcinoma were exposed.

Dihaloalkanes such as DCM and 1,2-dibromoethane can be activated by GSH conjugation catalyzed by GSTs to yield putative genotoxic intermediates (Guengerich, 2003). There is evidence suggesting that the hepatocarcinogenic effects of DCM in mice are associated with reactive GSH conjugates (via GST-T1) that bind to DNA (Sherratt et al., 2002). It was recently suggested that the occupational cholangiocarcinomas associated with exposure to 1,2-DCP might be attributed to 1,2-DCP acting as a carcinogen for bile duct cells though a GST-T1 catalyzed pathway as GST-T1 is constitutively expressed in normal human hepatocytes and bile duct epithelial cells (Sato et al., 2014). However, our unpublished data shows that there were no differences in the expression pattern of GST-T1 in livers between sporadic and 1,2-DCP-related occupational cholangiocarcinoma cases. In addition, 1,2DCP is not cholangiocarcinogenic to mice and rats, which like humans express GST-T1 in the cytoplasm of normal bile duct cells (Sato et al., 2014; Gi et al., 2015). In view of the protective effects of GST-T1-mediated glutathione conjugation against the toxicity of 1,2-DCP in hepatocytes and the fact that normal bile duct epithelial cells of hamsters do not express GST-T1 (Imberti et al., 1990; Gi et al., 2015), we expected that, compared to mice and rats, hamsters might be predisposed to the toxic effects of 1,2DCP in the bile duct. However, in the present study, no deleterious effects of 1,2-DCP were observed in bile duct epithelial cells in hamsters regardless of BOP-initiation. Currently, no deleterious effects of 1,2-DCP on bile duct epithelial cells have been observed in hamsters, mice, or rats irrespective of the expression pattern of GST-T1 in the bile duct epithelium, and therefore, the requirement for GST-T1 in the development of 1,2-DCP-related occupational cholangiocarcinomas remains unclear. As little is 
Effect of 1,2-DCP on BOP-induced cholangiocarcinogenesis in hamsters

yet known about the formation of DNA reactive metabolites derived from 1,2-DCP and their correlation with GSH-conjugates, further studies are needed to determine the metabolites of glutathione conjugated 1,2-DCP and compare the metabolic activity of GST-T1 toward 1,2DCP between experimental rodents and humans. Nevertheless, our findings that neither GST-T1 nor CYP2E1 was expressed in normal bile duct epithelium or atypical biliary hyperplasia regardless of 1,2-DCP treatment, suggests that 1,2-DCP had no effect on the expression of these two enzymes and that these enzymes were not involved in BOP-induced cholangiocarcinogenesis.

The present study indicates that 1,2-DCP lacks modifying effects on BOP-induced pancreatic carcinogenesis in hamsters as evidenced by the following facts: (1) In the 19-week experiment, regardless of 1,2-DCP treatment, pancreatic ductal carcinomas were observed in all the BOP-initiated groups and there were no significant differences among these groups. (2) While pancreatic ductal carcinomas were observed in groups treated with 1,2DCP after BOP initiation but not in the group given BOP initiation alone in the 17 -week experiment, this result is likely to be due to chance because when the total incidence and number of pancreatic ductal adenocarcinomas that occurred at weeks 17 and 19 were combined, there were no significant differences among the BOP-initiated groups (Table 3). (3) 1,2-DCP had no effect on the proliferative activity of atypical ductal hyperplasia or pancreatic ductal adenocarcinomas regardless of BOP initiation. (4) 1,2-DCP alone did not induce any proliferative lesions and had no effect on proliferative activity in the pancreatic duct epithelium in the 19-week experiment.

Treatment with 1,2-DCP by gavage induced hepatocellular carcinomas in mice but not in rats (NTP, 1986a), while inhalation exposure to 1,2-DCP induced nasal tumors in rats (Umeda et al., 2010). Moreover, treatment with 1,2-DCP by gavage did not exert carcinogenic effects in the present hamster study. These results indicate that the carcinogenic effects of 1,2-DCP are species- and exposure route-dependent and this is generally attributed to interspecies differences in metabolism, pharmacokinetics, and genetic background. It is well known that many chemicals that are carcinogenic in both experimental rodents and humans do not show cross-species/site concordance for specific tumor effect(s). Therefore, there is not sufficient evidence to dismiss a potential link of 1,2DCP to cholangiocarcinoma in humans, though at present there is no experimental evidence for 1,2-DCP cholangiocarcinogenicity.

In conclusion, the present study demonstrates that 1,2DCP lacks promoting effects on BOP-induced cholan- giocarcinogenesis and suggests the possibility that 1,2DCP is not carcinogenic to the hamster bile duct in the present model. To clarify the cholangiocarcinogenicity of 1,2-DCP in experimental animals, it will be necessary to determine the cholangiocarcinogenicity of concurrent exposure of 1,2-DCP with the other halogen solvents to which workers with cholangiocarcinomas were exposed, especially by inhalation exposure.

\section{ACKNOWLEDGMENTS}

The authors gratefully acknowledge the technical assistance of Rie Onodera, Keiko Sakata, Yuko Hisabayashi, Hideki Nakagawa and Yukiko Iura. This work was supported by a Grant-in-Aid for Scientific Research from Japan Society for the Promotion of Science (JSPS KAKENHI Grant Number 26340042), and Health Labour Sciences Research Grants (Research on Risk of Chemical Substances H26-\#25 and Research on Occupational Safety and Health H26-\#20) from the Ministry of Health, Labor and Welfare of Japan.

Conflict of interest---- The authors declare that there is no conflict of interest.

\section{REFERENCES}

Benbrahim-Tallaa, L., Lauby-Secretan, B., Loomis, D., Guyton, K.Z., Grosse, Y., El Ghissassi, F., Bouvard, V., Guha, N., Mattock, H., Straif, K. and International Agency for Research on Cancer Monograph Working, G. (2014): Carcinogenicity of perfluorooctanoic acid, tetrafluoroethylene, dichloromethane, 1,2dichloropropane, and 1,3-propane sultone. Lancet Oncol., 15, 924-925.

Blechacz, B. and Gores, G.J. (2008): Cholangiocarcinoma: advances in pathogenesis, diagnosis, and treatment. Hepatology, 48, 308-321.

Burek, J.D., Nitschke, K.D., Bell, T.J., Wackerle, D.L., Childs, R.C., Beyer, J.E., Dittenber, D.A., Rampy, L.W. and McKenna, M.J. (1984): Methylene chloride: a two-year inhalation toxicity and oncogenicity study in rats and hamsters. Fundam. Appl. Toxicol., 4, 30-47.

Furukawa, F., Nishikawa, A., Lee, I.S., Kanki, K., Umemura, T., Okazaki, K., Kawamori, T., Wakabayashi, K. and Hirose, M. (2003): A cyclooxygenase-2 inhibitor, nimesulide, inhibits postinitiation phase of N-nitrosobis(2-oxopropyl)amine-induced pancreatic carcinogenesis in hamsters. Int. J. Cancer, 104, 269273.

Gi, M., Fujioka, M., Yamano, S., Shimomura, E., Ishii, N., Kakehashi, A., Takeshita, M. and Wanibuchi, H. (2015): Determination of hepatotoxicity and its underlying metabolic basis of 1,2-dichloropropane in male Syrian hamsters and $\mathrm{B} 6 \mathrm{C} 3 \mathrm{~F} 1$ mice. Toxicol. Sci., 145, 196-208.

Guengerich, F.P. (2003): Activation of dihaloalkanes by thiol-dependent mechanisms. J. Biochem. Mol. Biol., 36, 20-27.

Hailey, J.R., Nold, J.B., Brown, R.H., Cullen, J.M., Holder, J.C., 
Jordan, H.L., Ennulat, D. and Miller, R.T. (2014): Biliary proliferative lesions in the Sprague-Dawley rat: adverse/non-adverse. Toxicol. Pathol., 42, 844-854.

IARC (2014): International Agency for Research on Cancer. Volume 110: Perfluoro-octanoic acid, tetrafl uoroethylene, dichloromethane, 1,2-dichloropropane, 1,3-propane sultone. IARC Working Group; Lyon, June 3-10, 2014. IARC Monogr. Eval. Carcinog. Risk Chem. Hum. (in press).

Imberti, R., Mapelli, A., Colombo, P., Richelmi, P., Bertè, F. and Bellomo, G. (1990): 1,2-Dichloropropane (DCP) toxicity is correlated with DCP-induced glutathione (GSH) depletion and is modulated by factors affecting intracellular GSH. Arch. Toxicol., 64, 459-465.

Imray, C.H., Newbold, K.M., Davis, A., Lavelle-Jones, M. and Neoptolemos, J.P. (1992): Induction of cholangiocarcinoma in the Golden Syrian hamster using methylazoxymethyl acetate. Eur. J. Surg. Oncol., 18, 373-378.

Kitahashi, T., Yoshimoto, M. and Imai, T. (2011): Novel immunohistochemical marker, integrin $\alpha(\mathrm{V}) \beta(3)$, for BOP-induced early lesions in hamster pancreatic ductal carcinogenesis. Oncol. Lett., 2, 229-234.

Klaunig, J.E. and Kamendulis, L.M. (2008): Chemical carcinogenesis. In Casarett and Doull's toxicology : the basic science of poisons. (Casarett, L.J., Doull, J. and Klaassen, C.D. eds.), pp.329334, McGraw-Hill, New York.

Kubo, S., Nakanuma, Y., Takemura, S., Sakata, C., Urata, Y., Nozawa, A., Nishioka, T., Kinoshita, M., Hamano, G., Terajima, H., Tachiyama, G., Matsumura, Y., Yamada, T., Tanaka, H., Nakamori, S., Arimoto, A., Kawada, N., Fujikawa, M., Fujishima, H., Sugawara, Y., Tanaka, S., Toyokawa, H., Kuwae, Y., Ohsawa, M., Uehara, S., Sato, K.K., Hayashi, T. and Endo, G. (2014): Case series of 17 patients with cholangiocarcinoma among young adult workers of a printing company in Japan. J. Hepatobiliary Pancreat. Sci., 21, 479-488.

Kumagai, S. (2014): Two offset printing workers with cholangiocarcinoma. J. Occup. Health, 56, 164-168.

Kumagai, S., Kurumatani, N., Arimoto, A. and Ichihara, G. (2013): Cholangiocarcinoma among offset colour proof-printing workers exposed to 1,2-dichloropropane and/or dichloromethane. Occup. Environ. Med., 70, 508-510.

Kurashina, M., Kozuka, S., Nakasima, N., Hirabayasi, N. and Ito, M. (1988): Relationship of intrahepatic bile duct hyperplasia to cholangiocellular carcinoma. Cancer, 61, 2469-2474.

Nakanuma, Y. (2012): Tutorial review for understanding of cholangiopathy. Int. J. Hepatol., 2012, 547840.

NTP (1986a): NTP Toxicology and Carcinogenesis Studies of 1,2Dichloropropane (Propylene Dichloride) (CAS No. 78-87-5) in F344/N Rats and B6C3F1 Mice (Gavage Studies). Natl. Toxicol. Program Tech. Rep. Ser., 263, 1-182.

NTP (1986b): NTP Toxicology and Carcinogenesis Studies of Dichloromethane (Methylene Chloride) (CAS No. 75-09-2) in F344/N Rats and B6C3F1 Mice (Inhalation Studies). Natl. Toxicol. Program Tech. Rep. Ser., 306, 1-208.

Okamura, T., Umemura, T., Inoue, T., Tasaki, M., Ishii, Y., Nakamura, Y., Park, E.Y., Sato, K., Matsuo, T., Okamoto, S., Nishikawa, A. and Ogawa, K. (2013): Chemopreventive effects of 4-methylthio-3-butenyl Isothiocyanate (Raphasatin) but not curcumin against pancreatic carcinogenesis in hamsters. J. Agric. Food Chem., 61, 2103-2108.

Pour, P.M., Runge, R.G., Birt, D., Gingell, R., Lawson, T., Nagel, D., Wallcave, L. and Salmasi, S.Z. (1981): Current knowledge of pancreatic carcinogenesis in the hamster and its relevance to the human disease. Cancer, 47, 1573-1589.

Pour, P.M., Salmasi, S.Z. and Runge, R.G. (1978): Selective induction of pancreatic ductular tumors by single doses of $\mathrm{N}$-nitrosobis(2-oxopropyl)amine in Syrian golden hamsters. Cancer Lett., 4, 317-323.

Sato, Y., Kubo, S., Takemura, S., Sugawara, Y., Tanaka, S., Fujikawa, M., Arimoto, A., Harada, K., Sasaki, M. and Nakanuma, Y. (2014): Different carcinogenic process in cholangiocarcinoma cases epidemically developing among workers of a printing company in Japan. Int. J. Clin. Exp. Pathol., 7, 47454754.

Serota, D.G., Thakur, A.K., Ulland, B.M., Kirschman, J.C., Brown, N.M., Coots, R.H. and Morgareidge, K. (1986a): A two-year drinking-water study of dichloromethane in rodents. I. Rats. Food Chem. Toxicol., 24, 951-958.

Serota, D.G., Thakur, A.K., Ulland, B.M., Kirschman, J.C., Brown, N.M., Coots, R.H. and Morgareidge, K. (1986b): A two-year drinking-water study of dichloromethane in rodents. II. Mice. Food Chem. Toxicol., 24, 959-963.

Sherratt, P.J., Williams, S., Foster, J., Kernohan, N., Green, T. and Hayes, J.D. (2002): Direct comparison of the nature of mouse and human GST T1-1 and the implications on dichloromethane carcinogenicity. Toxicol. Appl. Pharmacol., 179, 89-97.

Sobue, T., Utada, M., Makiuchi, T., Ohno, Y., Uehara, S., Hayashi, T., Kogawa Sato, K. and Endo, G. (2015): Risk of bile duct cancer among printing workers exposed to 1,2-dichloropropane and/ or dichloromethane. J. Occup. Health., 57, 230-236.

Takahashi, M., Hori, M., Mutoh, M., Wakabayashi, K. and Nakagama, H. (2011): Experimental animal models of pancreatic carcinogenesis for prevention studies and their relevance to human disease. Cancers (Basel), 3, 582-602.

Takahashi, M., Kitahashi, T., Ishigamori, R., Mutoh, M., Komiya, M., Sato, H., Kamanaka, Y., Naka, M., Maruyama, T., Sugimura, T. and Wakabayashi, K. (2008): Increased expression of inducible nitric oxide synthase (iNOS) in N-nitrosobis(2oxopropyl)amine-induced hamster pancreatic carcinogenesis and prevention of cancer development by ONO-1714, an iNOS inhibitor. Carcinogenesis, 29, 1608-1613.

Takahashi, M., Pour, P., Althoff, J. and Donnelly, T. (1977): The pancreas of the syrian hamster (Mesocricetus auratus). I Anatomical study. Lab. Anim. Sci., 27, 336-342.

Thamavit, W., Pairojkul, C., Tiwawech, D., Itoh, M., Shirai, T. and Ito, N. (1993): Promotion of cholangiocarcinogenesis in the hamster liver by bile duct ligation after dimethylnitrosamine initiation. Carcinogenesis, 14, 2415-2417.

Umeda, Y., Matsumoto, M., Aiso, S., Nishizawa, T., Nagano, K., Arito, H. and Fukushima, S. (2010): Inhalation carcinogenicity and toxicity of 1,2-dichloropropane in rats. Inhal. Toxicol., 22, 1116-1126. 